\title{
Rapid Measurement of I-V Curves via Complete Information Acquisition
}

\author{
S. Somnath ${ }^{1,2}$, P. Maksymovych ${ }^{1,2}$, S. V. Kalinin, ${ }^{1,2}$ S. Jesse ${ }^{1,2}$ and R. K. Vasudevan ${ }^{1,2}$ \\ 1. Center for Nanophase Materials Sciences, Oak Ridge National Laboratory, Oak Ridge TN \\ 2. Institute for Functional Imaging of Materials, Oak Ridge National Laboratory, Oak Ridge TN
}

Current-voltage (I-V) measurements are perhaps the oldest spectroscopic technique in scanning probe microscopy. The measurement of $\mathrm{I}-\mathrm{V}$ (as well as derivatives $\mathrm{dI} / \mathrm{dV}$, etc.) has been instrumental in revealing surface states, electronic structures, quasiparticle interference patterns, and electronic modulations on surfaces of materials with nanoscale (and even atomic) resolution for decades. The key advantage is the ability to measure the properties as a function of spatial position, which is typically undertaken by dividing the area of interest into a spectroscopic array (grid) of points, and then positioning the SPM tip at each of these grid sites for the experiment. The main drawback to most spectroscopic techniques, however, remains the time taken for acquisition. In fact, the most basic of all measurements - the acquisition of a current-voltage (I-V) curve in SPM remains extremely slow, typically taking several seconds per measurement. Here, we show a new method based on complete information acquisition to increase this rate by $100-1000 x$.

We first note that in the most basic configuration, the aim of the experiment is to determine the resistance of the material $R$ under test as a function of the applied voltage $V$. Given that there are parasitic capacitances in the circuit (tip-sample capacitance, cables, etc.), these will also influence the measurement of the current $I$. The circuit equation reads $\mathrm{I}=\mathrm{V} / \mathrm{R}+\mathrm{CdV} / \mathrm{dt}$, where $V$ is the applied voltage, and $C$ is the capacitance of the measurement system. For this equation, $I-V$ measurements typically attempt to minimize the contribution of the second term by adding a delay time $t_{1}$ after the voltage is changed, typically on the order of a few ms. This is highly successful at eliminating noise and the parasitic capacitance contribution, but severely limits acquisition rates. Here we instead propose to apply a fast AC waveform, as opposed to the DC sweep, as shown in Fig. 1(a,b). The AC excitation to the tip is at 200Hz, and we simply measure the current without use of any delay or integration times, effectively creating an $\mathrm{RC}$ circuit problem where capacitance is unknown. Results are shown in Fig. 1(c,d) and Fig. 1(e-h), for a sample of $\mathrm{LaMnO}_{3}$ in ultra-high vacuum conditions. The fast I-V measurement, termed 'general-mode' I$\mathrm{V}$ (and an addition to a suite of general-mode SPM [1] techniques) allows for I-V curves to be acquired at scanning rates; in this case, yielding 100,000 curves in 17 minutes. An example of a point measurement from the scan is shown in Fig. 1(d), and clearly indicates that the I-V curves are beset by a large capacitance contribution.

Attempts have been made previously to attempt to determine the capacitance in such circuits. [2-4] Here, we provide a simple alternative based on the presence of regions of low conductivity in the sample. By utilization of k-means clustering, we can isolate the regions of the sample where the current flow is minimal, and essentially probe the limit equation (1) when $\mathrm{R} \rightarrow \infty$. This allows us to directly estimate the capacitance, and therefore recover the true resistance and current without the influence of the parasitic capacitance. These results are indicated in Fig. 2(c).

The results shown here show that for samples with regions of varying conductivity, it may be possible to infer the capacitance. In other cases, where the data is smooth, direct convolution of equation (1) to infer $\mathrm{R}$ is possible, if one assumes that the value of the current in the limit $\mathrm{V} \rightarrow 0$ is dominated by the second term. In the future, more sophisticated methods such as Bayesian inference could be utilized to infer the 
form of the resistance and the capacitance terms, which could be further improved via physical constraints on the form of $R$, such as positivity and monotonicity, and provide uncertainty quantification during the reconstruction [5].

\section{References:}

[1] A Belianinov, SV Kalinin, and S Jesse, Nat. Commun. 6 (2015) p. 6650

[2] R Meyer et al., Appl. Phys. Lett. 86 (2005), p. 142907

[3] S Martin et al., Rev. Sci. Instr. 88 (2017), p. 023901

[4] Y Komoto et al., Scientific Reports 6 (2016), p. 26606

[5] This research was sponsored by the Division of Materials Sciences and Engineering, BES, DOE (RKV, SVK, PM, SS). This research was conducted and partially supported (SJ) at the Center for Nanophase Materials Sciences, which is a US DOE Office of Science User Facility.
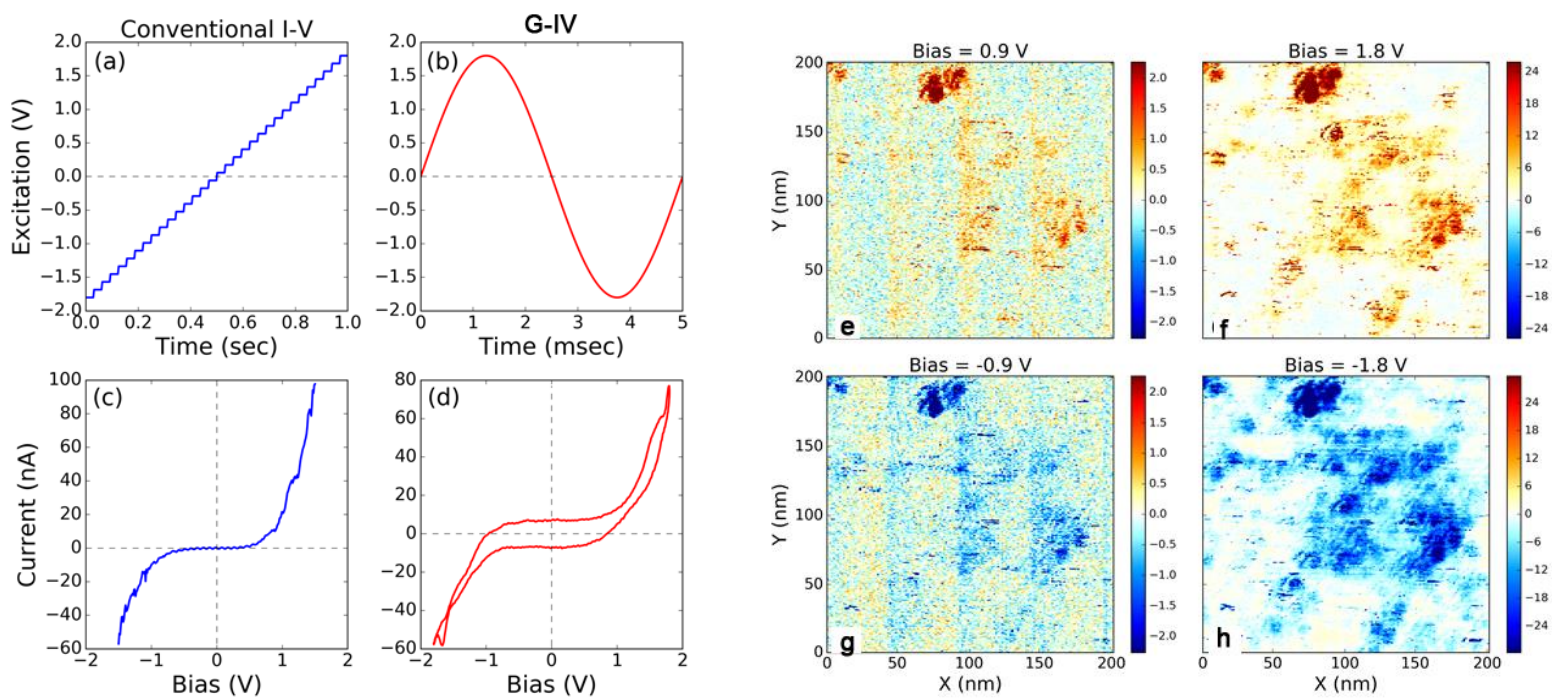

Figure 1. Comparison of standard I-V and G-IV applied to epitaxial thin film of $\mathrm{LaMnO}_{3}$ grown on (001) $\mathrm{SrTiO}_{3}$. Single point results from (a,c) Standard I-V measurement and (b,d) G-IV measurement. (e-h) Spatial maps from G-IV experiment of the current (nA).
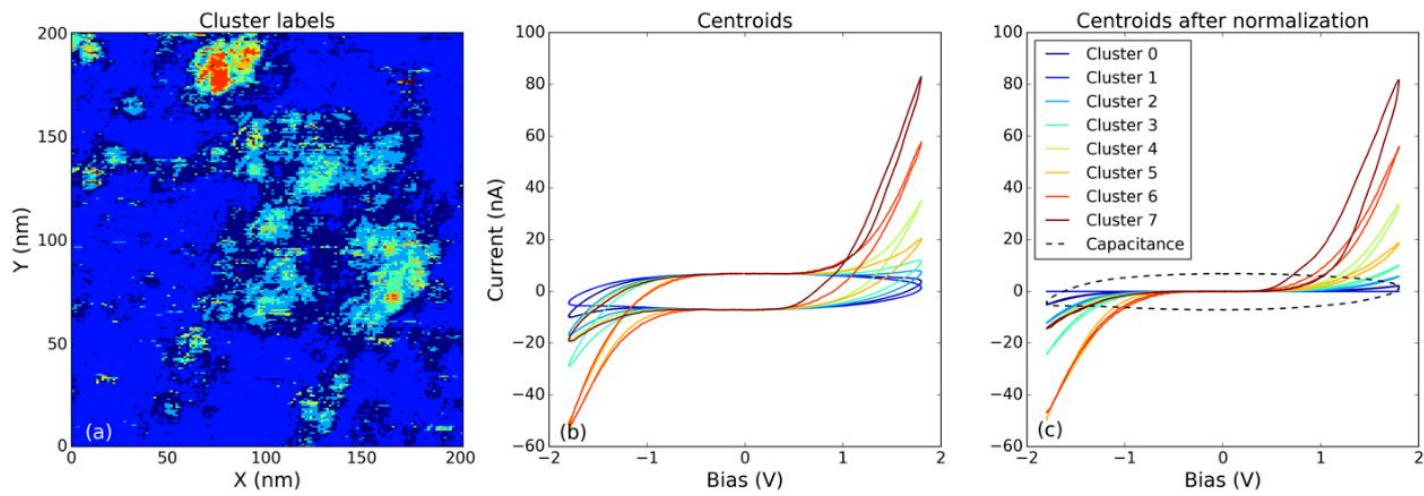

Figure 2. K-means cluster analysis of G-IV dataset. (a) cluster label map and (b) Centroids. (c) Centroids after removal of the capacitance contribution. 